Monatsschr Kinderheilkd 2010 - 158:736-737 DOI 10.1007/s00112-010-2198-0

Online publiziert: 6 . August 2010

(c) Springer-Verlag 2010

F. Zepp

Zentrum für Kinder- und Jugendmedizin, Universitätsmedizin Mainz

\title{
Chronisch-entzündliche Darmerkrankungen
}

punkthefts zu stellen. Erstmals greifen wir in dieser Ausgabe auch wichtige Aspekte der Überleitung pädiatrischer Patienten in die Betreuung durch internistische Gastroenterologen auf. Die Kinderund Jugendgastroenterologie stellt zudem einen Programmschwerpunkt des Jahreskongresses der DGKJ in Potsdam im September dieses Jahres dar.

S. Koletzko und H. H. Uhlig vom Dr. von Haunerschen Kinderspital des Klinikums der Universität München diskutieren die Bedeutung von Umwelteinflüssen auf die Entstehung und den Verlauf von CED. Nachvollziehbar erläutern sie das Konzept der Hygienehypothese, die postuliert, dass unter dem Einfluss von stark wechselnden Umgebungsbedingungen die Inzidenzen von Krankheiten wie Allergien, Autoimmunerkrankungen und CED in den vergangenen Jahrzehnten deutlich zugenommen haben. Veränderungen des Lebensstils (weniger Kontakt zu Nutztieren, Rauchen), verbesserte sanitäre und medizinische Maßnahmen (Nutzung von Antibiotika und Anthelminthika) sowie Änderungen unserer Ernährungsgewohnheiten (Stillen, „fast food“) spielen hierbei eine wesentliche Rolle. Das Verständnis der immunologischen Konsequenzen von Parasitenbesiedlung, Nutztierkontakt oder Stillen könnte helfen, zukünftig Maßnahmen zur Immunmodulation für Therapie, aber auch Prävention der CED zu entwickeln.

Der Beitrag von S. Buderus vom St.Marien-Hospital in Bonn befasst sich mit der Epidemiologie und den klinischen Besonderheiten von CED bei Kindern und Jugendlichen. Aus der Analyse epidemiologischer Daten ist es möglich, Rückschlüsse auf krankheitsauslösende Faktoren und die Pathophysiologie der CED zu gewinnen. Das ,pädiatrische Altersmaximum" der CED liegt bei 12 bis 14 Jahren, etwa $60 \%$ der Patienten leiden an einem MC und rund $30 \%$ an einer CU. Die Colitis indeterminata (CI) wird in der Pädiatrie häufiger diagnostiziert als in der „Erwachsenenmedizin", wobei insbesondere jüngere Patienten betroffen sind. Aktuelle CED-Inzidenzen liegen zwischen 4 und 1o Neuerkrankungen pro Jahr und 100.00o Menschen jünger als 18 Jahre; es scheint unverändert einen langsamen weiteren Anstieg zu geben. Der Autor erläutert zudem die Bedeutung von CED-Erkrankungsregistern und der Anwendung diagnostischer Klassifikationen, die die Qualität der Datenerfassung und die Vergleichbarkeit der Studien erhöhen.

Biologika, insbesondere TNFa-Blocker, haben in den vergangenen Jahren die Therapie von CED nachhaltig optimiert. M. Radke von der Klinik für Kinder- und Jugendmedizin des Klinikums Ernst von Bergmann in Potsdam beschreibt in seinem Beitrag die Wirkungsweise und die Einsatzmöglichkeiten dieser neuen Gruppe von biologischen Arzneimitteln, die gezielt immunologische Mechanismen der Krankheitsentstehung und -ausbreitung beeinflussen.

\section{(7) Biologika, insbesondere TNFa-Blocker, haben in den vergangenen Jahren die Therapie nachhaltig optimiert}

Infliximab ist als bisher einziger TNFaBlocker für Kinder mit moderatem bzw. schwerem MC zugelassen. Studiendaten belegen einen steroidsparenden und das körperliche Wachstum optimierenden Effekt. Bei Versagen einer Standardtherapie wird Infliximab zunehmend früher 
im Therapiekonzept eingesetzt. Auch für Kinder mit schwerer CU stellt Infliximab trotz fehlender Zulassung eine Therapieoption dar. Bei Wirkungsverlust von Infliximab kann der Einsatz weiterer neuer Biologika nach Aufklärung der Eltern und Patienten sowie Vorliegen des Einverständnisses erwogen werden.

M. Classen von der Klinik für Kinderund Jugendmedizin des Klinikums Links der Weser in Bremen erläutert in seinem Artikel Indikationen und Verfahren der nichtmedikamentösen Therapieverfahren bei Kindern mit CED. Der Fokus dieses Beitrags liegt auf Ernährungsinterventionen und den Indikationen von chirurgischen Eingriffen. Wichtig für die langfristige Betreuung der Patienten mit MC ist, dass die enterale Ernährungstherapie mit flüssiger Formelnahrung die Chance bietet, ohne relevantes Nebenwirkungsrisiko eine klinische Remission zu induzieren bzw. zu unterstützen.

Chirurgische Eingriffe bei CED im Kindesalter sind vor allem bei Patienten mit Komplikationen oder mit einem therapieresistenten Verlauf indiziert. So kann bei schwer verlaufender CU durch Kolektomie eine Heilung der Grunderkrankung erreicht werden, allerdings mit relevanten postoperativen Risiken wie z. B. einer Pouchitis. Beim MC werden chirurgische Therapieverfahren vor allem bei Strikturen und bei Fisteln eingesetzt.

Moderne Diagnostik und Therapie haben die Lebensqualität von Patienten mit chronischen Erkrankungen in den vergangenen Jahrzehnten eindrucksvoll verbessert. Dies gilt auch für Kinder und Jugendliche mit CED, die bei konsequenter pädiatrischer Betreuung heute in der Regel in sehr guter körperlicher Verfassung das junge Erwachsenalter erreichen. Umso bedeutender ist der zielgerichtete Übergang (Transition) des Jugendlichen oder jungen Erwachsenen mit einer CED vom kind- zum erwachsenenzentrierten medizinischen Versorgungssystem. K. M. Keller von der Deutschen Klinik für Diagnostik in Wiesbaden beschreibt in seinem Beitrag nicht nur den Weg von Kindern und Jugendlichen mit CED in das Erwachsenenalter, sondern erläutert auch Hindernisse auf Seiten der Patienten und ihrer Familien wie auch der beteiligten Therapeuten. Entscheidend für das Ge- lingen dieses Prozesses sind die Initiative des Kindergastroenterologen, angepasst an die körperliche, seelische und emotionale Reife des Jugendlichen, und eine langfristig gemeinsam erarbeitete Bereitschaft von Patient, Eltern und medizinischem Team.

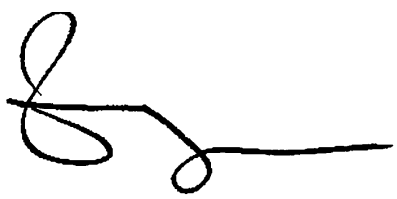

Prof. Dr. F. Zepp

\section{Korrespondenzadresse \\ Prof. Dr. F. Zepp}

Zentrum für Kinder- und Jugendmedizin, Universitätsmedizin Mainz Langenbeckstraße 1, 55131 Mainz Zepp@kinder.klinik.uni-mainz.de

\section{NCL-Forschungspreis}

Die Neuronale Zeroidlipofuszinose (NCL) ist die häufigste neurodogenerative Krankheit bei Kindern. Diese unheilbare Stoffwechselerkrankung führt unweigerlich zum Tode des Patienten. Die erbliche Krankheit verläuft progressiv und führt zu Erblindung, Demenz, epileptischen Anfällen, dem Verlust der Sprachfähigkeit und Paralyse. Bundesweit sind etwa 400 Kinder davon betroffen, weltweit zählt man etwa 50.000 erkrankte Kinder. Aufgrund der Seltenheit der Krankheit kommt es häufig zu Fehldiagnosen.

Die NCL-Stiftung lobt jährlich den NCL-Forschungspreis in Höhe von 50.000 Euro aus. Ziel dieses Preises ist es, die Erforschung dieser seltenen Krankheit juvenile NCL, die weltweit noch nicht ausreichend gefördert wird, anhand beispielhafter Projekte voranzutreiben.

Besonders preiswürdig sind innovative klinisch-orientierte Projekte. Das Preisgeld soll für ein Doktorandenstipendium eingesetzt werden, um das eingereichte Projekt konkret umzusetzen und den wissenschaftlichen Nachwuchs gezielt zu fördern.

Es werden weltweit Forscher/innen aufgefordert, Projekte einzureichen, um zur Heilung der juvenilen NCL beizutragen. Wissenschaftler/innen aus verwandten Gebieten wie Alzheimer, Altern und lysosomale Speicherkrankheiten werden ermutigt, einen Antrag zu stellen.

Quelle und weitere Informationen: www.ncl-stiftung.de 